\title{
An Efficient Semi-analytical Framework for Micromechanical Modeling of Transverse Cracks in Fiber-reinforced Composites
}

\author{
Evan J. Pineda* and Anthony M. Waas ${ }^{\dagger}$ \\ University of Michigan, Ann Arbor, MI 48109
}

\author{
Brett A. Bednarcyk $\ddagger$ \\ NASA Glenn Research Center, Cleveland, $\mathrm{OH} 44135$
}

Craig S. Collier $\S$

Collier Research Corporation, Newport News, VA 23607

\begin{abstract}
The growth of transverse cracks in a composite layer is investigated using the semi-analytical high-fidelity generalized method of cells to represent a repeating unit cell of the composite. This method discretizes the domain into a number of subcells, each of which is occupied by a constituent of the composite. A non-linear displacement field approximation is used within each subcell, and displacement continuity and traction continuity are enforced, on average, at each subcell interface. Discontinuities are introduced at the subcell interfaces through the evolving compliance interface model. In this model traction and separation are related via a time dependent parameter. The predicted response for two simple examples of composite micro-architectures is presented and discussed.
\end{abstract}

\section{Introduction}

Q Uantitative characterization of failure mechanisms in a progressively damaging fiber reinforced composite material (FRCM) can be difficult to achieve experiments alone. Experiments provide powerful insight into failure mechanisms and, in some cases, high volumes of testing alone can become time consuming and expensive. Virtual testing is a valuable complement to physical testing of composite materials. ${ }^{1}$ Simulation of large-scale, composite structures can be performed, providing insight that can aid in the design of a structure made of FRCM. Additionally, virtual testing allows any number of designs to be considered when physical testing of the same number of designs would become impractical. Moreover, simulations can be used to observe intricate details, in real time, about damage and failure evolution within a structure that may not be available in an experiment.

Virtual tests can also be utilized at a lesser scale. The numerous and highly interactive damage mechanisms that arise in composites can be difficult to isolate. Moreover, these mechanisms evolve across multiple length scales. Simulations can be used to resolve the damage mechanisms at the appropriate scale. Additionally, multiscale simulations can be used to tie the scales together. ${ }^{2-7}$

The focus of this paper is to demonstrate an efficient, virtual framework for simulating transverse crack growth in uni-directional, fiber-reinforced laminates (FRLs) at the micro-level. Numerous micromechanical models have been developed that can capture the details at the microscale. ${ }^{8-12}$ The method employed herein is the high-fidelity generalized method of cells (HFGMC), an extension of the original generalized method of cells (GMC) ${ }^{10}$ developed by Aboudi et. al. ${ }^{13}$ This method divides a repeating unit cell (RUC), that is representative of the composite microstructure, into a number of material subcells. Higher-order polynomial are used to approximate the displacement fields in the subcells. Displacement and traction continuity are enforced the subcell boundaries to determine the local fields in terms of the applied global fields. The constitutive behavior of the subcell materials is general and numerous constitutive theories can be applied. Aboudi et. al demonstrated the use of HFGMC with inelastic phases in Ref. 14, and Bednarcyk et al. utilized HFGMC to model progressive damage within an RUC in Ref.15.

The present work models transverse crack growth at the micro-scale as interfacial debonding at matrix-matrix and fiber-matrix interfaces within an HFGMC RUC. The Micromechanics Analysis Code with Generalized Method of

\footnotetext{
*Ph.D Candidate, Mechanical Engineering Department.

$\dagger$ Professor, Aerospace and Mechanical Engineering Departments.

${ }^{\ddagger}$ Materials Research Engineer, AIAA Senior Member.

$\S$ President, Hypersizer Software, AIAA Senior Member
} 
Cells (MAC/GMC) Suite of Micromechanics Codes, developed at the NASA Glenn Research Center (GRC), serves as the computational platform for the analysis presented here. ${ }^{16,17}$ The evolving compliant interface model (ECI) is currently integrated into MAC/GMC to capture interfacial debonding. This model was used in conjunction with HFGMC to capture fiber-matrix debonding in metal matrix composites in Ref. 18. The ECI model is explicitly dependent on time; thus, it is not ideal for modeling fracture. However, the purpose of this paper is to demonstrate that the framework is in place to perform detailed, micromechanical analysis of transverse crack growth in FRLs. Efforts are currently under way to incorporate the discrete cohesive zone method (DCZM) ${ }^{19-22}$ into MAC/GMC. This would enable simulations that allow traction-separation laws to govern the interfacial debonding and fracture.

\section{The High-Fidelity Generalized Method of Cells}

HFGMC is based on a homogenization technique for composites with periodic microstructure as shown in Figure 1(a) in terms of the global coordinates $\left(x_{1}, x_{2}, x_{3}\right)$. The parallelepiped repeating unit cell, Figure 1(b), defined with respect to local coordinates $\left(y_{1}, y_{2}, y_{3}\right)$, of such a composite is divided into $N_{\alpha}, N_{\beta}$ and $N_{\gamma}$ subcells in the $y_{1}, y_{2}$ and $y_{3}$ directions, respectively. Each subcell is labeled by the indices $(\alpha \beta \gamma)$ with $\alpha=1, \ldots, N_{\alpha}, \beta=1, \ldots, N_{\beta}$ and $\gamma=1, \ldots, N_{\gamma}$, and may contain a distinct homogeneous material. The dimensions of subcell $(\alpha \beta \gamma)$ in the $y_{1}, y_{2}$ and $y_{3}$ directions are denoted by $d_{\alpha}, h_{\beta}$ and $l_{\gamma}$, respectively. A local coordinate system $\left(\bar{y}_{1}^{(\alpha)}, \bar{y}_{2}^{(\beta)}, \bar{y}_{3}^{(\gamma)}\right)$ is introduced in each subcell whose origin is located at its center.

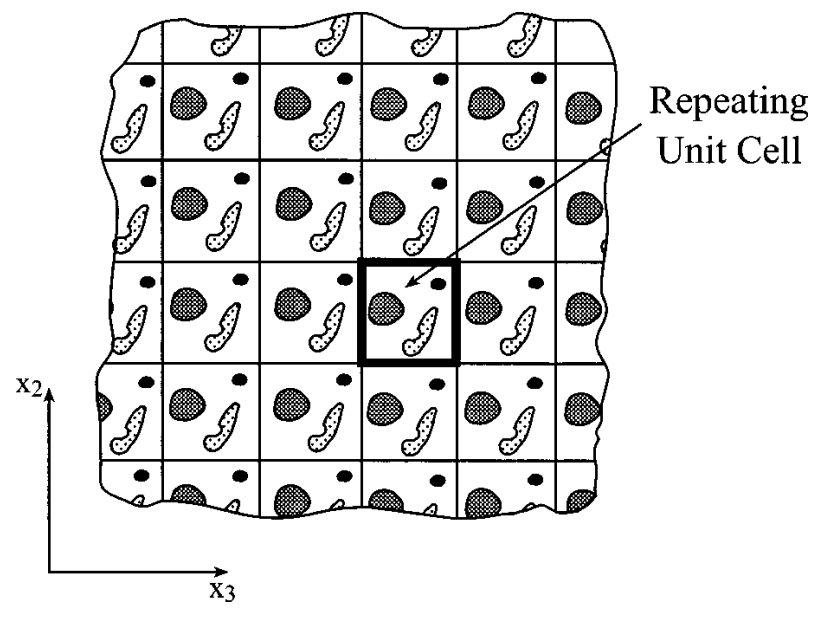

(a) Multiphase composite with repeating microstructure. ${ }^{14}$

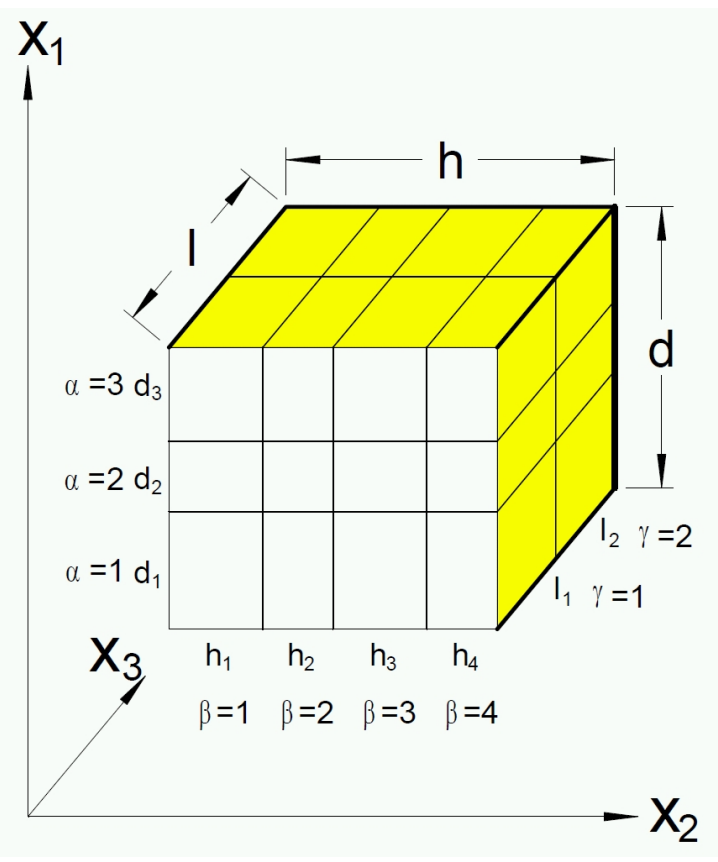

(b) RUC used in HFGMC. ${ }^{16}$

Figure 1. by

The local (subcell) constitutive equation of the material which, in general, is assumed to be thermoinelastic is given

$$
\boldsymbol{\sigma}^{(\alpha \beta \gamma)}=\mathbf{C}^{(\alpha \beta \gamma)}\left(\boldsymbol{\epsilon}^{(\alpha \beta \gamma)}-\boldsymbol{\epsilon}^{I(\alpha \beta \gamma)}\right)-\boldsymbol{\Gamma}^{(\alpha \beta \gamma)} \Delta T
$$

where $\boldsymbol{\sigma}^{(\alpha \beta \gamma)}, \boldsymbol{\epsilon}^{(\alpha \beta \gamma)}, \boldsymbol{\epsilon}^{I(\alpha \beta \gamma)}$ and $\boldsymbol{\Gamma}^{(\alpha \beta \gamma)}$ are the stress, total strain, inelastic strain and thermal stress tensors, respectively, in subcell $(\alpha \beta \gamma)$. In Equation (1), $\mathbf{C}^{(\alpha \beta \gamma)}$ is the stiffness tensor of the material in the subcell $(\alpha \beta \gamma)$, and $\Delta T$ denotes the temperature deviation from a reference temperature. The inelastic strain $\epsilon^{I(\alpha \beta \gamma)}$ is governed either by the Prandtl-Reuss equations of the classical plasticity or by an appropriate viscoplastic flow rule.

The basic assumption in HFGMC is that the displacement vector $\mathbf{u}^{(\alpha \beta \gamma)}$ in each subcell is expanded into quadratic 
forms in terms of its local coordinates $\left(\bar{y}_{1}^{(\alpha)}, \bar{y}_{2}^{(\beta)}, \bar{y}_{3}^{(\gamma)}\right)$ as follows.

$$
\begin{aligned}
\mathbf{u}^{(\alpha \beta \gamma)} & =\overline{\boldsymbol{\epsilon}} \mathbf{x}+\mathbf{W}_{(000)}^{(\alpha \beta \gamma)}+\bar{y}_{1}^{(\alpha)} \mathbf{W}_{(100)}^{(\alpha \beta \gamma)}+\bar{y}_{2}^{(\beta)} \mathbf{W}_{(010)}^{(\alpha \beta \gamma)}+\bar{y}_{3}^{(\gamma)} \mathbf{W}_{(001)}^{(\alpha \beta \gamma)} \\
& +\frac{1}{2}\left(3 \bar{y}_{1}^{(\alpha) 2}-\frac{d_{\alpha}^{2}}{4}\right) \mathbf{W}_{(200)}^{(\alpha \beta \gamma)}+\frac{1}{2}\left(3 \bar{y}_{2}^{(\beta) 2}-\frac{h_{\beta}^{2}}{4}\right) \mathbf{W}_{(020)}^{(\alpha \beta \gamma)} \\
& +\frac{1}{2}\left(3 \bar{y}_{3}^{(\gamma) 2}-\frac{l_{\gamma}^{2}}{4}\right) \mathbf{W}_{(002)}^{(\alpha \beta \gamma)}
\end{aligned}
$$

where $\overline{\boldsymbol{\epsilon}}$ is the applied (external) average strain, and the unknown terms $\mathbf{W}_{(l m n)}^{(\alpha \beta \gamma)}$, must be determined from the fulfillment of the equilibrium conditions, the periodic boundary conditions, and the interfacial continuity conditions of displacements and tractions between subcells. The periodic boundary conditions ensure that the displacements and tractions at opposite surfaces of the repeating unit cell are identical, see Ref. 14 for more details. A principal ingredient in the present micromechanical analysis is that all these conditions are imposed in the average (integral) sense.

As a result of the imposition of the equilibrium equations in the subcells together with the application of the interfacial and periodicity conditions, a linear system of algebraic equations is obtained which can be represented in the following form

$$
\mathbf{K U}=\mathbf{f}+\mathbf{g}
$$

where the matrix $\mathbf{K}$ contains information on the geometry and thermomechanical properties of the materials within the individual subcells $(\alpha \beta \gamma)$, and the displacement vector $\mathbf{U}$ contains the unknown displacement coefficient $\mathbf{W}_{(\operatorname{lmn})}^{(\alpha \beta \gamma)}$ which appear on the right-hand side of Equation (1). The mechanical vector $\mathbf{f}$ contains information on the applied average strains $\bar{\epsilon}$ and the imposed temperature deviation $\Delta T$. The inelastic force vector $\mathbf{g}$ appearing on the right-hand side of Equation (3) contains the inelastic effects given in terms of the integrals of the inelastic strain distributions. These integrals depend implicitly on the elements of the displacement coefficient vector $\mathbf{U}$, requiring an incremental solution procedure, of the $21 N_{\alpha} N_{\beta} N_{\gamma}$ algebraic equations in Equation (3), at each point along the loading path. To obtain the full details of Equation (3) and the solution procedure please refer to Ref. 14.

The solution of Equation (3) enables the establishment of the following localization relation which expresses the average strain $\overline{\boldsymbol{\epsilon}}^{(\alpha \beta \gamma)}$ in the subcell $(\alpha \beta \gamma)$ to the externally applied average strain $\overline{\boldsymbol{\epsilon}}$ in the form:

$$
\overline{\boldsymbol{\epsilon}}^{(\alpha \beta \gamma)}=\mathbf{A}^{(\alpha \beta \gamma)} \overline{\boldsymbol{\epsilon}}+\mathbf{A}^{t h(\alpha \beta \gamma)} \Delta T+\mathbf{D}^{I(\alpha \beta \gamma)}
$$

where $\mathbf{A}^{(\alpha \beta \gamma)}$ and $\mathbf{A}^{t h(\alpha \beta \gamma)}$ are the mechanical and thermal strain concentration tensors, respectively, of the subcell $(\alpha \beta \gamma)$, and $\mathbf{D}^{I(\alpha \beta \gamma)}$ is a vector that involves the current inelastic effects in the subcell.

The final form of the effective constitutive law of the multiphase thermo-inelastic composite, which relates the average stress $\bar{\sigma}$ and strain $\bar{\epsilon}$, is established as follows:

$$
\overline{\boldsymbol{\sigma}}=\mathbf{C}^{*} \overline{\boldsymbol{\epsilon}}-\left(\boldsymbol{\Gamma}^{*} \Delta T+\overline{\boldsymbol{\sigma}}^{I}\right)
$$

In this equation $\mathbf{C}^{*}$ is the effective stiffness tensor and $\boldsymbol{\Gamma}^{*}$ is the effective thermal stress tensor of the composite, and $\bar{\sigma}^{I}$ is the global inelastic stress tensor. All these global quantities can be expressed in a closed-form manner in terms of the mechanical and thermal concentration tensors which appear in Eq. (4) together with the inelastic term $\mathbf{D}^{I(\alpha \beta \gamma)}$, see Ref. 14 for more details. They are given as follows:

$$
\begin{gathered}
\mathbf{C}^{*}=\frac{1}{D H L} \sum_{\alpha=1}^{N_{\alpha}} \sum_{\beta=1}^{N_{\beta}} \sum_{\gamma=1}^{N_{\gamma}} d_{\alpha} h_{\beta} l_{\gamma} \mathbf{C}^{(\alpha \beta \gamma)} \mathbf{A}^{(\alpha \beta \gamma)} \\
\boldsymbol{\Gamma}^{*}=\frac{-1}{D H L} \sum_{\alpha=1}^{N_{\alpha}} \sum_{\beta=1}^{N_{\beta}} \sum_{\gamma=1}^{N_{\gamma}} d_{\alpha} h_{\beta} l_{\gamma}\left[\mathbf{C}^{(\alpha \beta \gamma)} \mathbf{A}^{t h(\alpha \beta \gamma)}-\boldsymbol{\Gamma}^{(\alpha \beta \gamma)}\right]
\end{gathered}
$$

and

$$
\overline{\boldsymbol{\sigma}}^{I}=\frac{-1}{D H L} \sum_{\alpha=1}^{N_{\alpha}} \sum_{\beta=1}^{N_{\beta}} \sum_{\gamma=1}^{N_{\gamma}} d_{\alpha} h_{\beta} l_{\gamma}\left[\mathbf{C}^{(\alpha \beta \gamma)} \mathbf{D}^{I(\alpha \beta \gamma)}-\mathbf{R}_{(0,0,0)}^{(\alpha \beta \gamma)}\right]
$$

where $\mathbf{R}_{(0,0,0)}^{(\alpha \beta \gamma)}$ is an expression that represents the integral of the inelastic strain distributions. 


\section{Evolving Compliant Interface Model}

The ECI model has been used previously to simulate displacement discontinuities within the GMC and HFGMC micromechanics frameworks. ${ }^{18,23}$ This approach follows the basic form proposed by Jones and Whittier ${ }^{24}$ where the discontinuity in displacement across an interface is proportional to the traction at that interface,

$$
\left[u_{j}\right]^{I}=\left.R_{j}(t) \sigma_{j}\right|^{I} ;\left.\quad e_{D B}\right|^{I} \geq 1
$$

where $\left[u_{j}\right]^{I}$ is the jump in displacement in the direction $j$ (normal, shear or tangential) across interface $I,\left.\sigma^{j}\right|^{I}$ is the traction in the direction $j$ at the interface. The requirement for interfacial debonding $\left.e_{D B}\right|^{I}$ is similar to the Hashin-Rotem failure criteria, ${ }^{25}$

$$
\left.e_{D B}\right|^{I}=\left(\frac{\left.\sigma_{n}\right|^{I}}{\left.\sigma_{n}^{C}\right|^{I}}\right)^{2}+\left(\frac{\left.\sigma_{s}\right|^{I}}{\left.\sigma_{s}^{C}\right|^{I}}\right)^{2}+\left(\frac{\left.\sigma_{t}\right|^{I}}{\left.\sigma_{t}^{C}\right|^{I}}\right)^{2}
$$

where $\left.\sigma_{j}^{C}\right|^{I}$ are critical interfacial strengths and $n, s$, and $t$ indicate normal, shear and tangential modes, or mode I, mode II, and mode III. ${ }^{26}$ Thus, when Equation (10) is satisfied debonding in all three directions initiates.

The proportionality constant $R_{j}$ represents the compliance of the interface. If $R_{j}$ is an implicit function of the traction, then Equation (9) can be thought of as a traction-separation law.

However, ECI invokes explicit dependence of $R_{j}$ on time,

$$
R_{j}(t)=\Lambda_{j}\left[\exp \left(\frac{t-t_{D B}}{B_{j}}\right)-1\right] ; t \geq t_{D B}
$$

where $t_{D B}$ is the time at which debonding initially occurs, and $\Lambda_{j}$ and $B_{j}$ are parameters that characterize the timedependent behavior of the interface. These parameters do not have a strict physical basis and cannot be measured, but rather were introduced to control the time evolution of $R_{j}$. When modeling matrix fracture, the proportionality parameter that relates the interfacial traction to the jump in displacement across the interface should be a function of either traction or displacement. Implementation of such relationships into MAC/GMC is currently underway, and ECI is used in this paper to demonstrate that HFGMC is an appropriate platform to model discontinuities at the micro-level in the form of transverse crack growth.

\section{Examples of Transverse Crack Growth in an RUC using HFGMC and ECI}

\section{IV.A. Single-fiber RUC}

The first example that will be used to demonstrate the application of discontinuous methods within a mircomechanics framework to capture the effects of transverse crack growth in the matrix phase of a fiber-reinforced composite is a single fiber RUC. The RUC, shown in Figure 2, contains a total of 38 x 38 subcells (520 fiber and 924 matrix subcells), with a $60 \%$ fiber volume fraction. The $x_{2}-x_{3}$ plane is the plane of transverse isotropy, and the $x_{1}$-direction is aligned with the fiber axis. Displacement discontinuities across each matrix-matrix interface and fiber-matrix interface are modeled using ECI. The same ECI parameters are used for both matrix-matrix and fiber-matrix interfaces in this investigation, but different values can be used. The RUC is subjected to transverse tensile strain, over a duration of 100 timesteps, under generalized plane strain conditions and periodic boundaries. The total simulation time was 3 min. $50 \mathrm{sec}$. A summary of RUC size and runtime is given in Table 1.

The transverse strain versus transverse stress response is presented in Figure 3. It is seen that the transverse stress quickly diminishes to zero subsequent to reaching the peak load. This indicates that transverse crack progression in this RUC is rapid.

The development of transverse cracks, modeled as subcell interfacial discontinuities using the ECI model, in the RUC are shown at various times in Figure 4. Interfaces at which debonding has initiated (satisfaction of Equation (10)) are marked in red in Figure 4. The first interfaces that begin to debond are lie on or adjacent to the fiber boundary. These cracks proceed to grow, and other crack begin to nucleate, perpendicular to the applied strain direction. The cracks propagate across the periodic boundary and essentially bridge adjacent fibers.

The transverse stresses $\sigma_{22}$ corresponding to the cracked RUCs in Figure 4 are displayed in Figure 5. Additionally the in-plane shear stresses $\tau_{23}$ in the RUC are plotted at the same times in Figure 6. Localization of $\sigma_{22}$ is evident in Figure 5 as the damage evolves in the RUC. Similarly, the coupling shear and normal interfacial debonding, imposed by Equation (10), is displayed in the $\tau_{23}$ plots. 


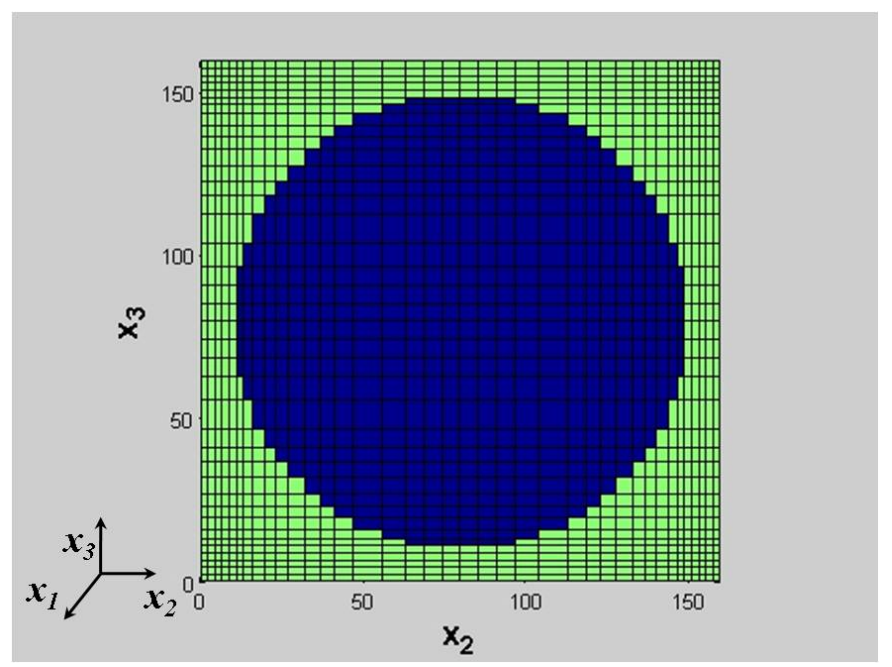

Figure 2. Single fiber RUC containing 32 x 32 subcells ( 520 fiber subcells and 924 subcells).

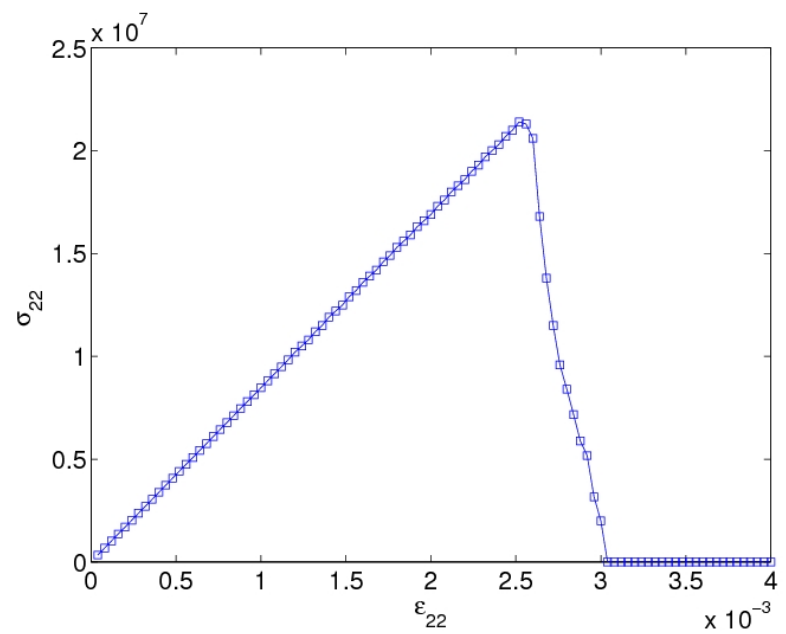

Figure 3. Transverse stress versus transverse strain for single fiber RUC containing $38 \times 38$ subcells. 


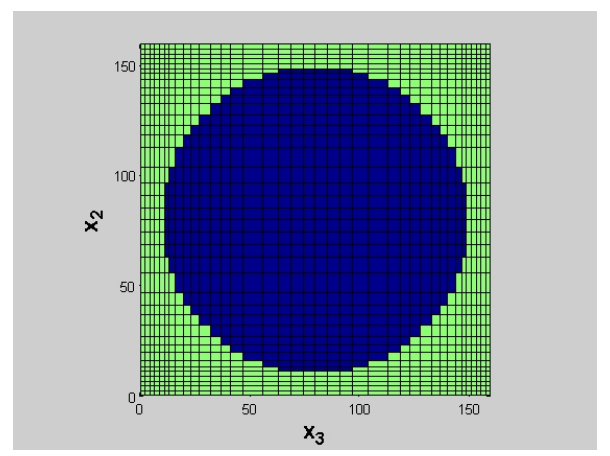

(a) $t=0.61$

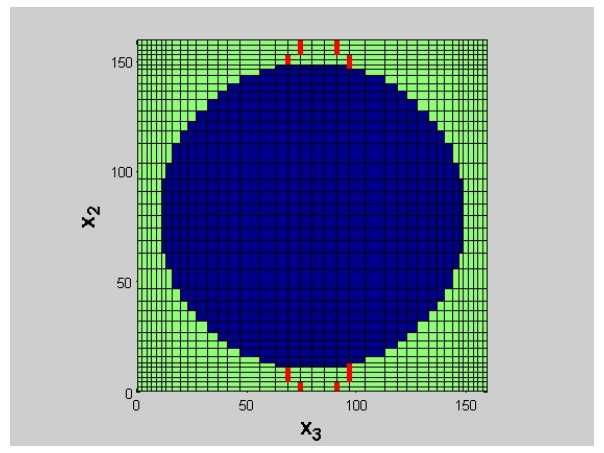

(c) $t=0.63$

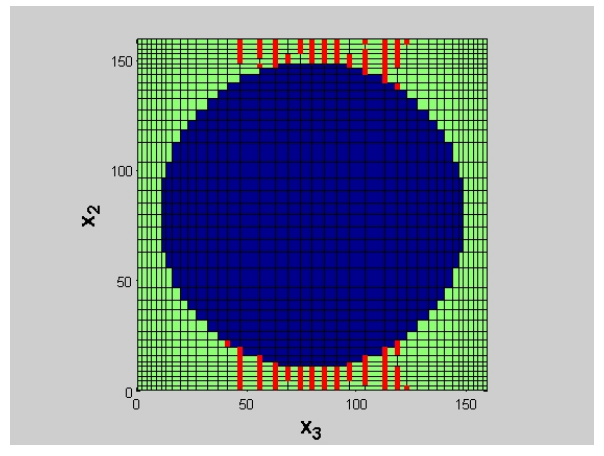

(e) $t=0.66$

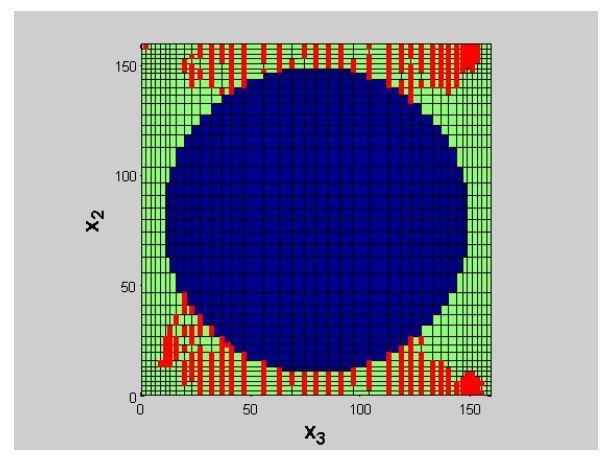

(g) $t=0.72$

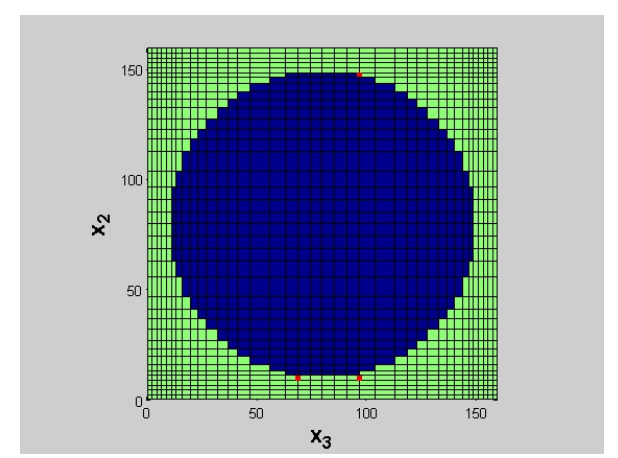

(b) $t=0.62$

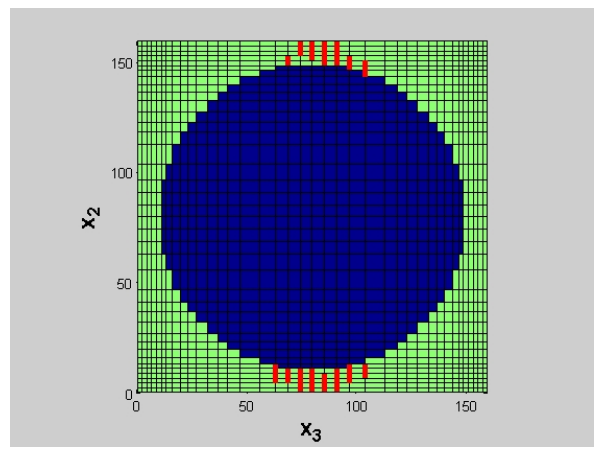

(d) $t=0.64$

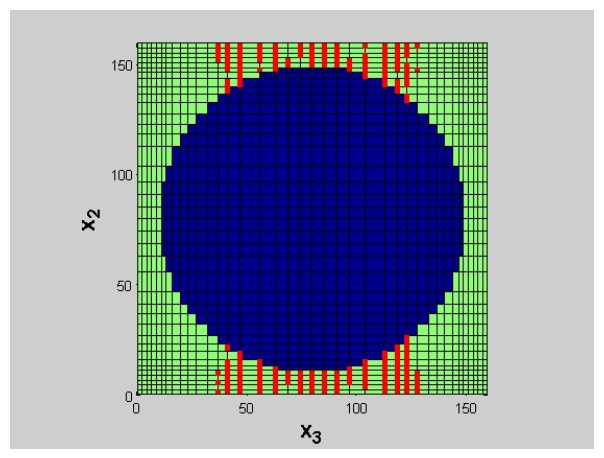

(f) $t=0.67$

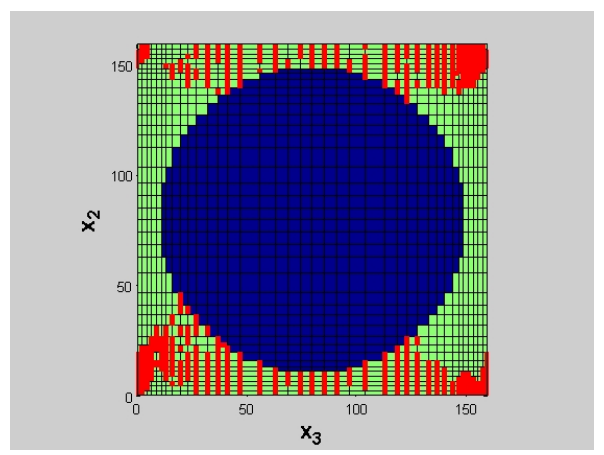

(h) $t=0.74$

Figure 4. Transverse crack evolution in single fiber RUC

6 of 13 


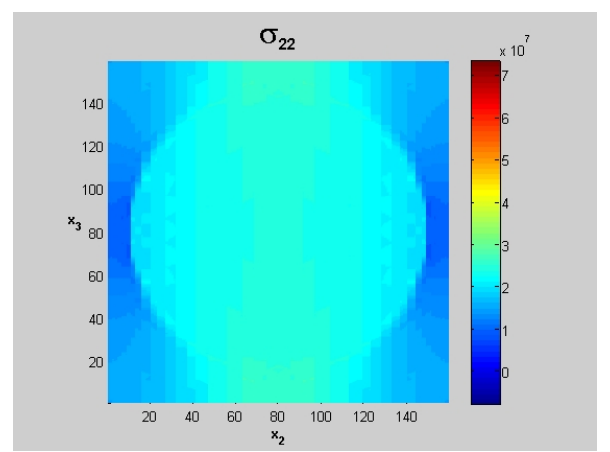

(a) $t=0.61$

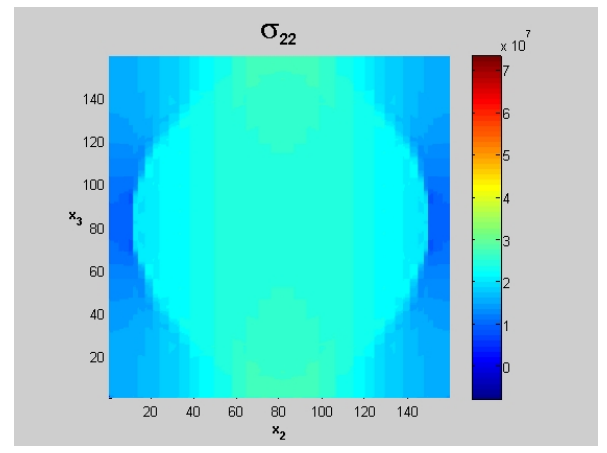

(c) $t=0.63$

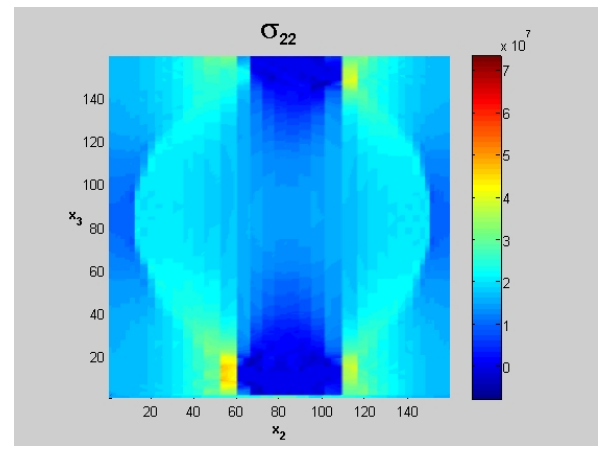

(e) $t=0.66$

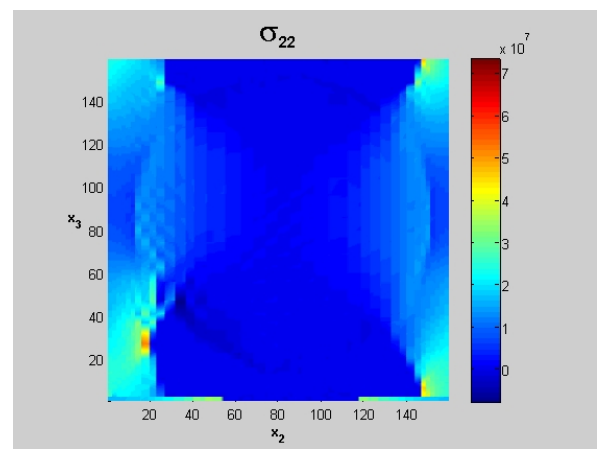

(g) $t=0.72$

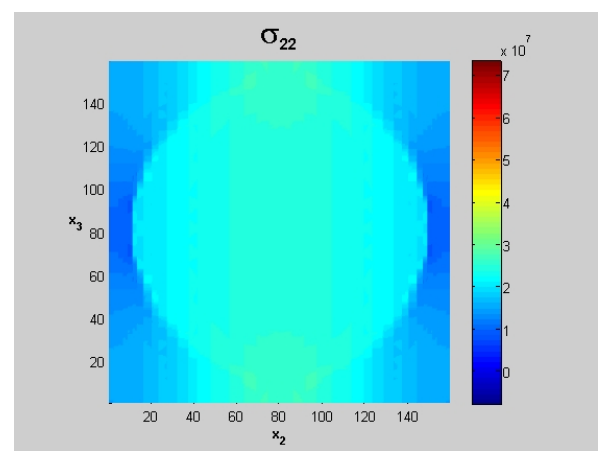

(b) $t=0.62$

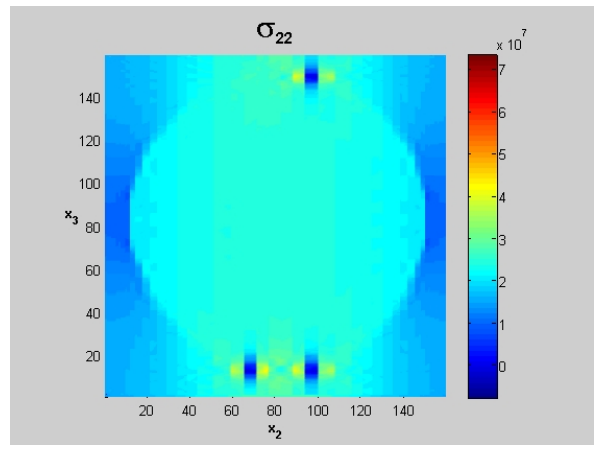

(d) $t=0.64$

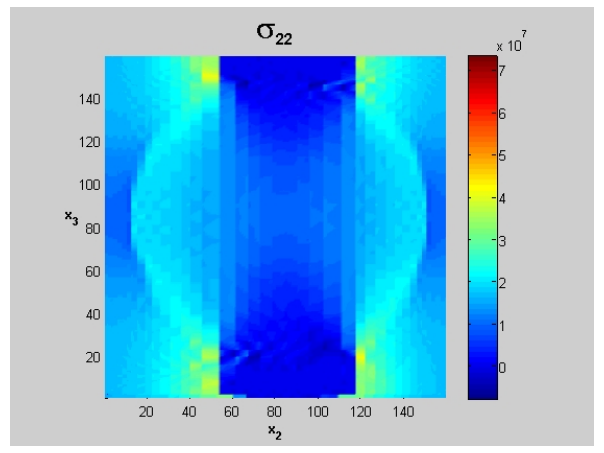

(f) $t=0.67$

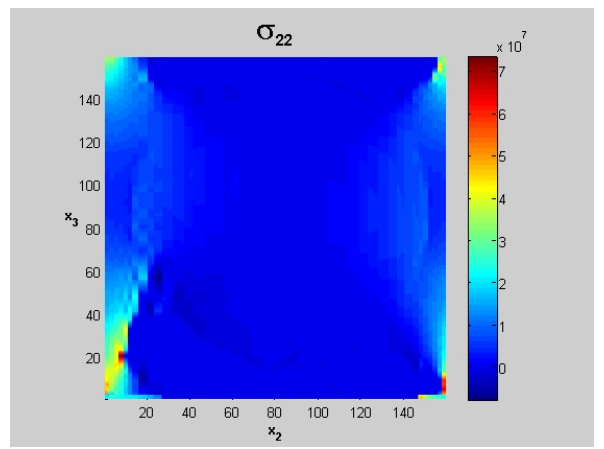

(h) $t=0.74$

Figure 5. Transverse stress $\left(\sigma_{22}\right)$ evolution in single fiber RUC 


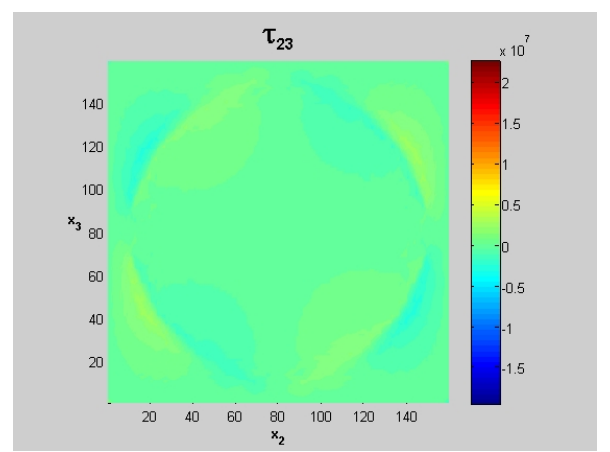

(a) $t=0.61$

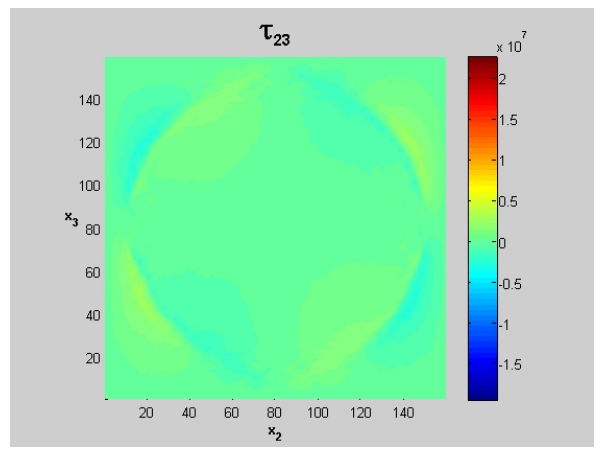

(c) $t=0.63$

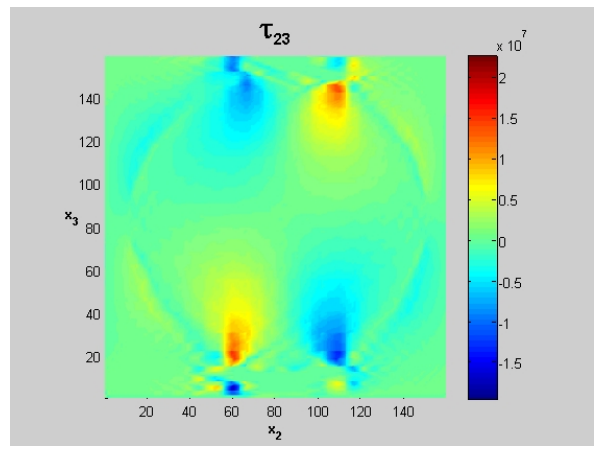

(e) $t=0.66$

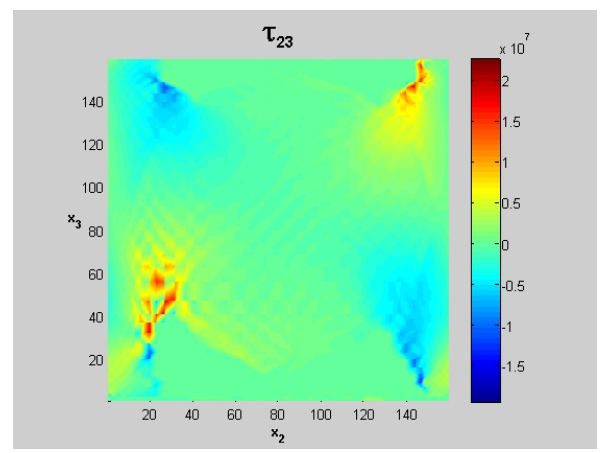

(g) $t=0.72$

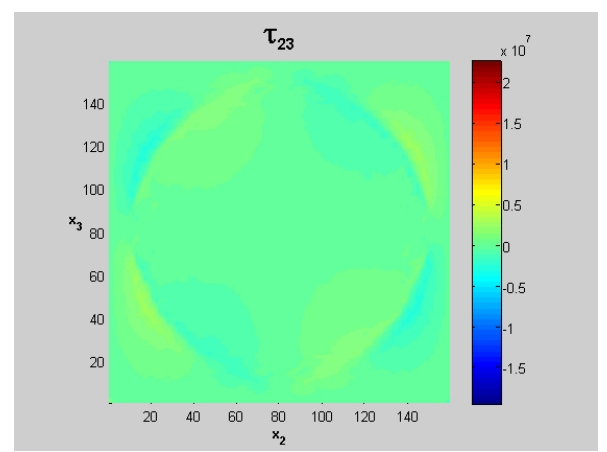

(b) $t=0.62$

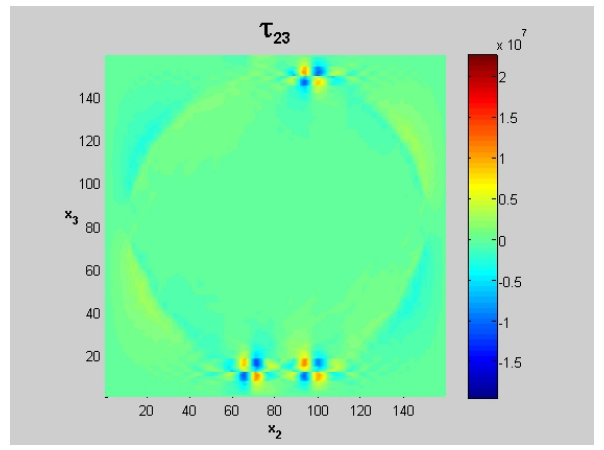

(d) $t=0.64$

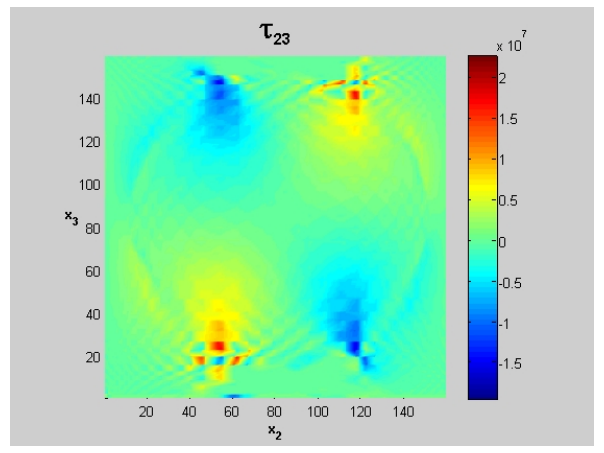

(f) $t=0.67$

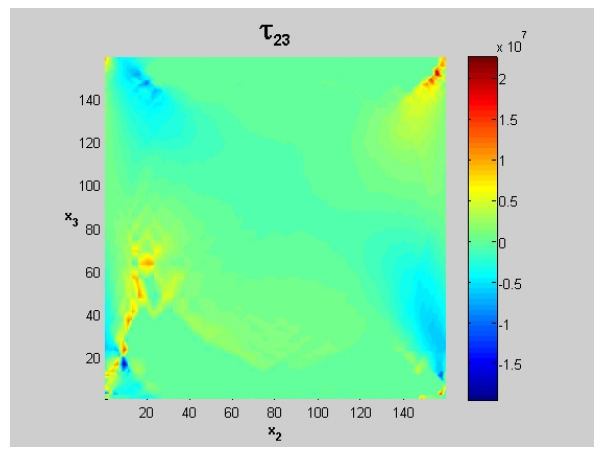

(h) $t=0.74$

Figure 6. Transverse stress $\left(\tau_{23}\right)$ evolution in single fiber RUC 


\section{IV.B. Two diagonal fibers}

The RUC used in the second example is shown in Figure 7. This RUC contains two fibers arranged diagonally. The $60 \%$ fiber volume fraction from the previous example is not preserved, but this architecture can more clearly demonstrate crack bridging between two fibers. The total size of the RUC was 76 x 76 subcells (1040 fiber subcells and 4736 matrix subcells). This RUC is also subjected to transverse tensile strain loading, over 100 timesteps. The total simulation time was $23 \mathrm{~min}$. and $42 \mathrm{sec}$. Runtime and RUC details are presented in Table 1. Figure 8 shows $\sigma_{22}$ vs. $\epsilon_{22}$. Similar, catastrophic failure is exhibited as in the single RUC example.

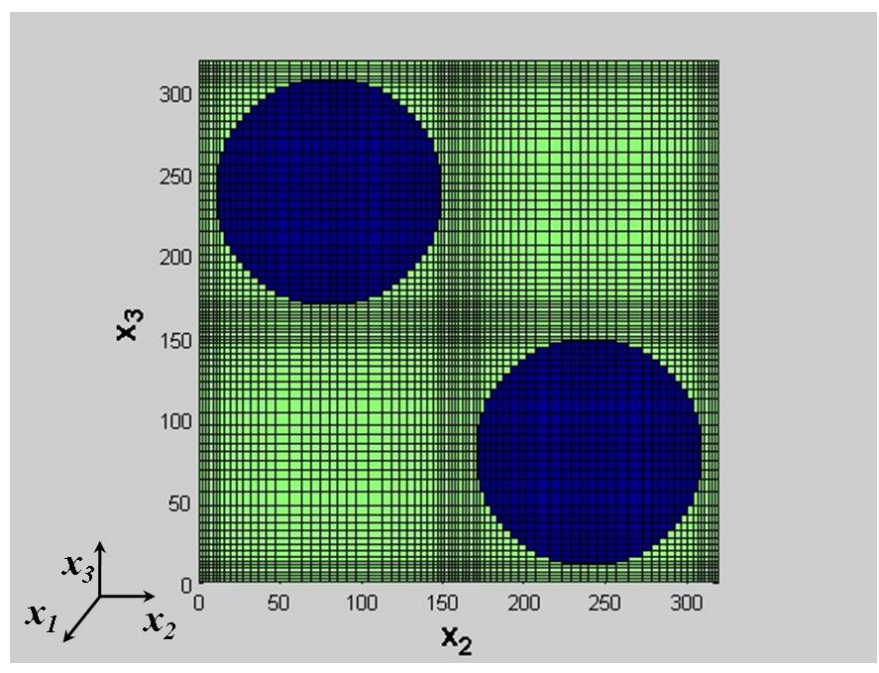

Figure 7. RUC containing two diagonal fibers and $76 \times 76$ subcells ( 1040 fiber subcells and 4736 subcells).

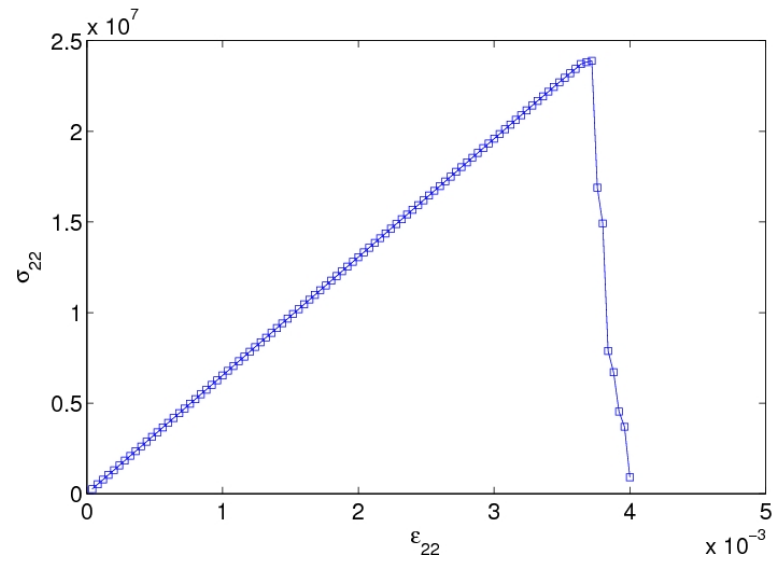

Figure 8. Transverse stress versus transverse strain for RUC containing two diagonal fibers and 76 x 76 subcells.

Transverse crack evolution is given in Figure 9. Again, crack growth initiates at the fiber-matrix interface. A large number of cracks nucleate from the fiber boundary. Eventually the cracks propagating from two fiber boundaries meet in the middle of the RUC and bridge the fibers. Figures 10 and 11 show the progression of $\sigma_{22}$ and $\tau_{12}$, respectively.

\begin{tabular}{|c|c|c|c|c|c|c|}
\hline RUC & Fiber Subcells & Matrix Subcells & Total Subcells & Debonding Interfaces & Timesteps & Runtime (sec.) \\
\hline \hline Single Fiber & 520 & 924 & 1,444 & 1,904 & 100 & 230 \\
\hline Two Diagonal Fibers & 1,040 & 4,736 & 5,776 & 9,584 & 100 & 1,422 \\
\hline
\end{tabular}

Table 1. Summary of RUC details and runtime for two example architectures. 


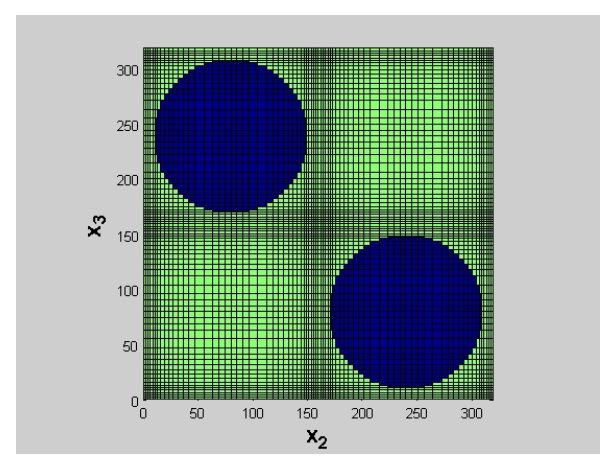

(a) $t=0.81$

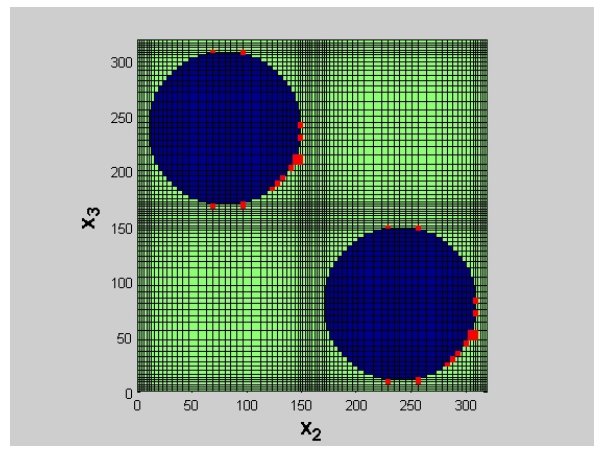

(c) $t=0.91$

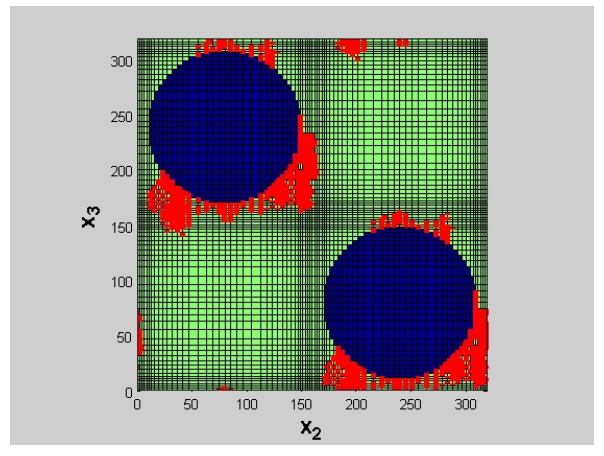

(e) $t=0.94$

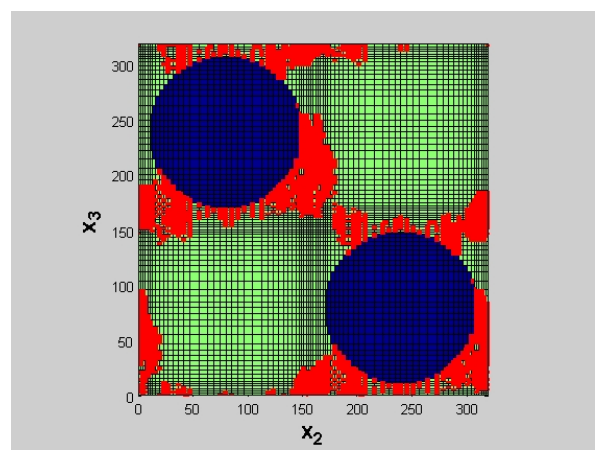

(g) $t=0.98$

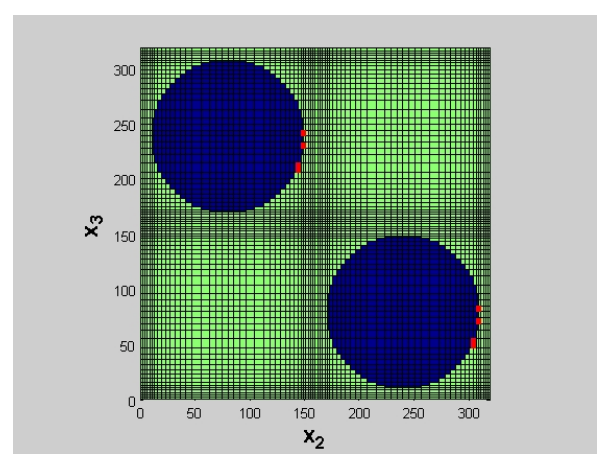

(b) $t=0.86$

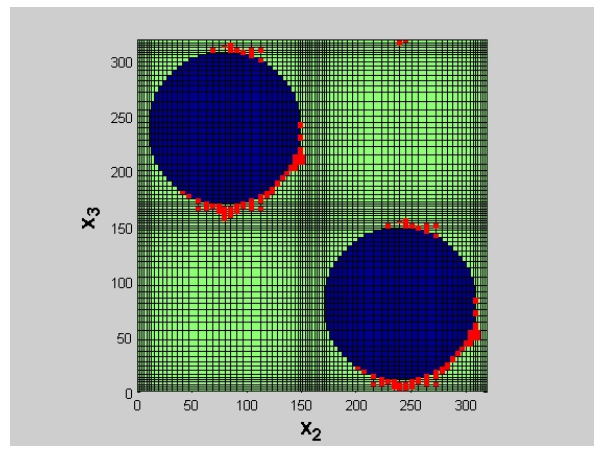

(d) $t=0.92$

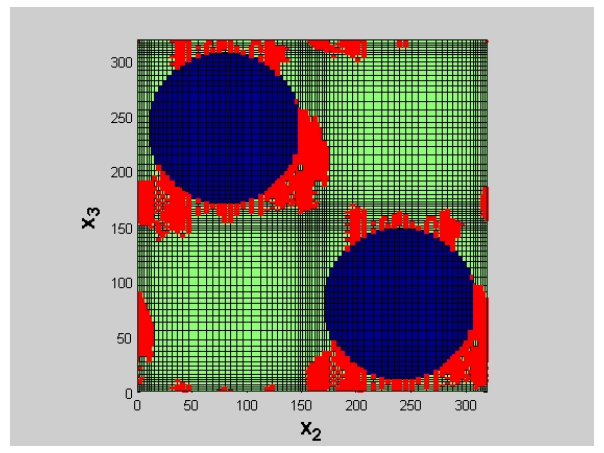

(f) $t=0.96$

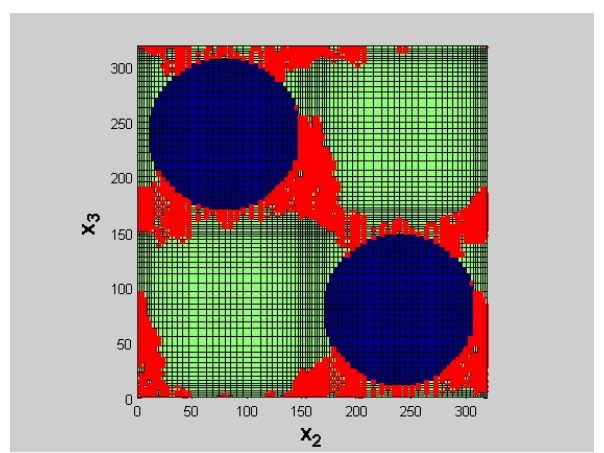

(h) $t=1.00$

Figure 9. Transverse crack evolution in RUC containing two diagonal fibers

10 of 13 


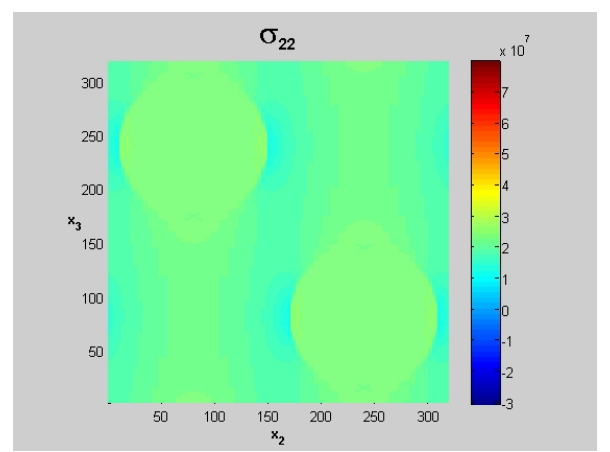

(a) $t=0.81$

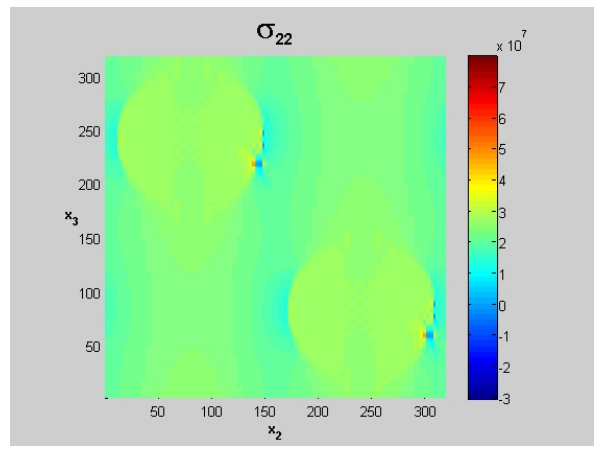

(c) $t=0.91$

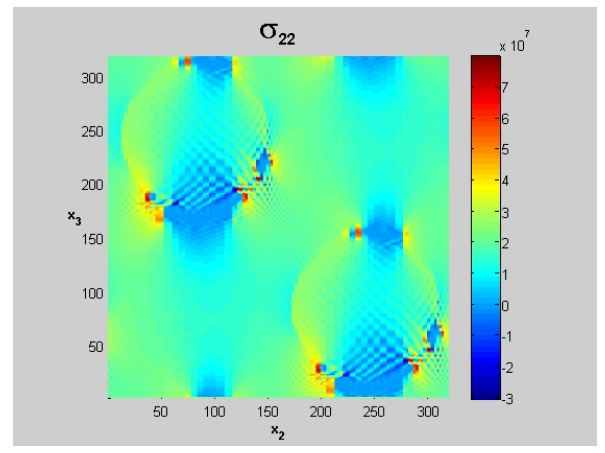

(e) $t=0.94$

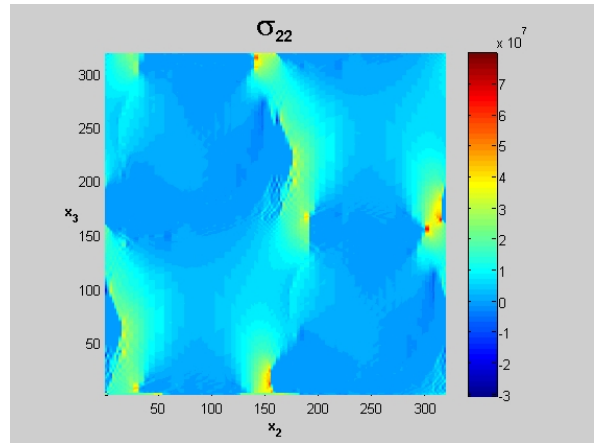

(g) $t=0.98$

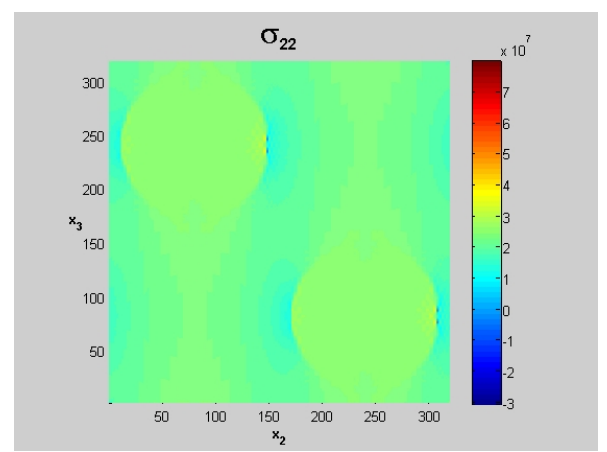

(b) $t=0.86$

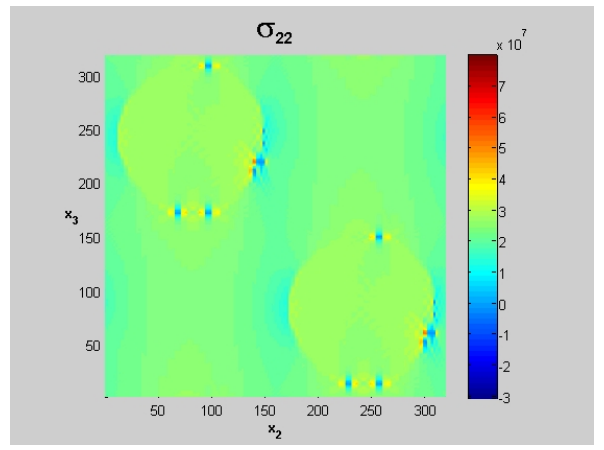

(d) $t=0.92$

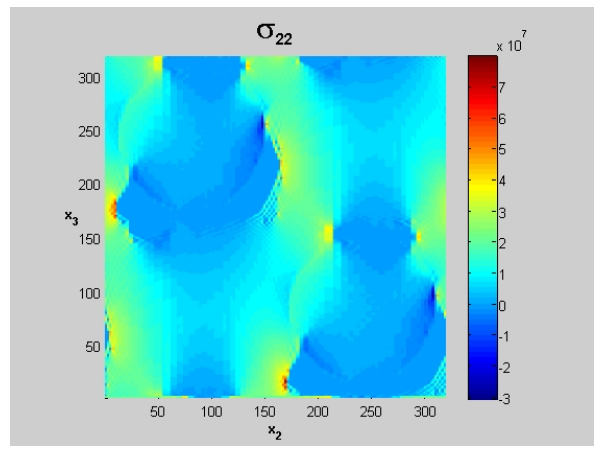

(f) $t=0.96$

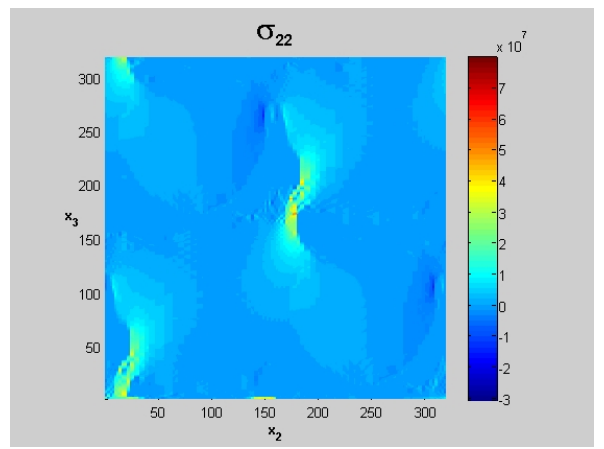

(h) $t=1.00$

Figure 10. Transverse stress $\left(\sigma_{22}\right)$ evolution in RUC containing two diagonal fibers

11 of 13 


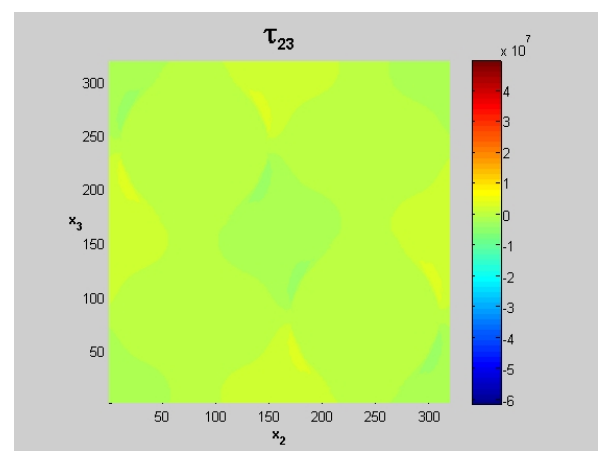

(a) $t=0.81$

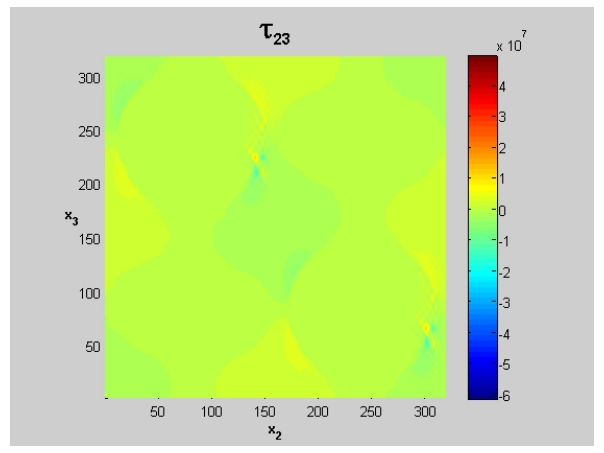

(c) $t=0.91$

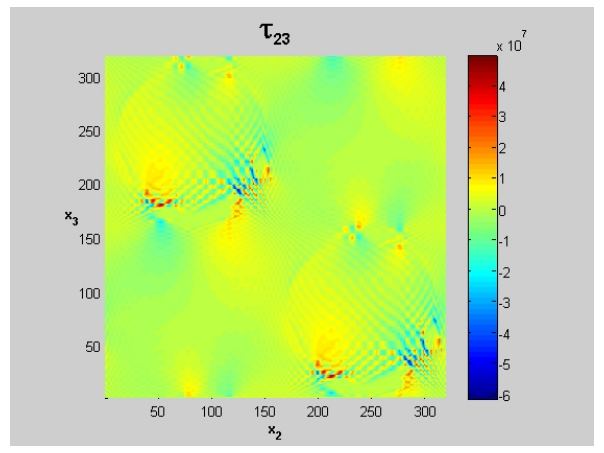

(e) $t=0.94$

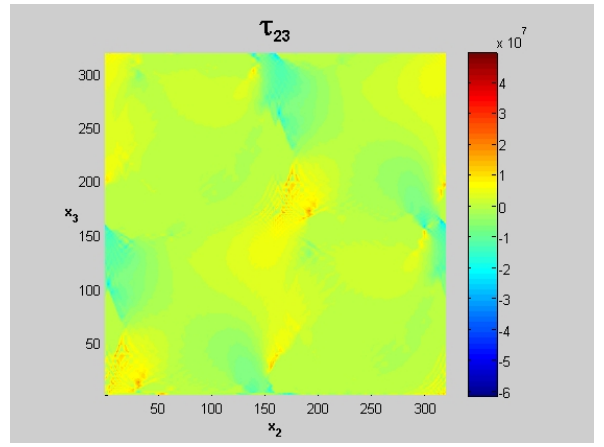

(g) $t=0.98$

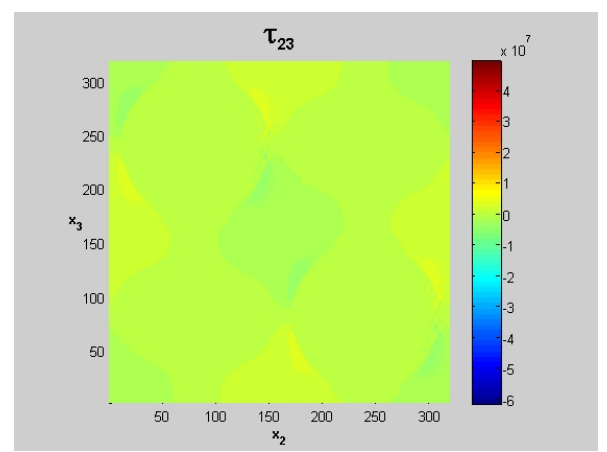

(b) $t=0.86$

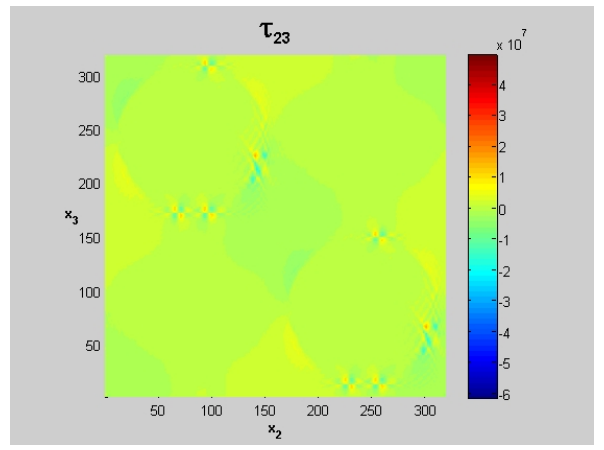

(d) $t=0.92$

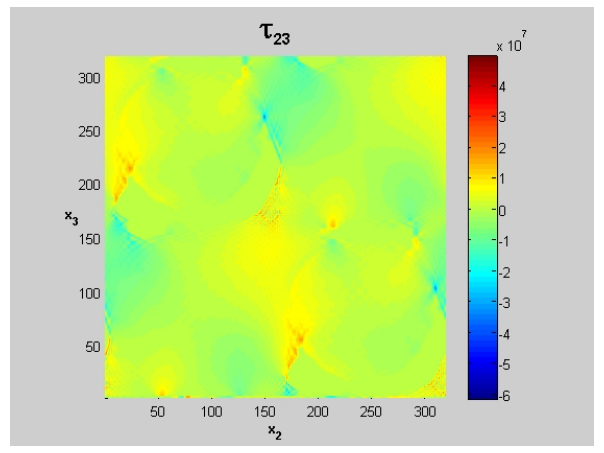

(f) $t=0.96$

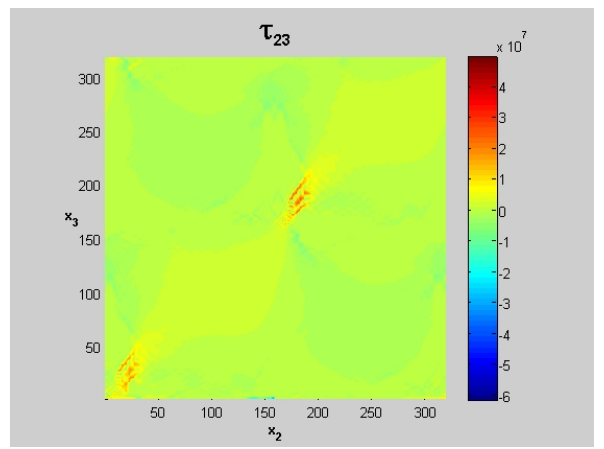

(h) $t=1.00$

Figure 11. Transverse stress $\left(\tau_{23}\right)$ evolution in RUC containing two diagonal fibers 


\section{Conclusions}

A micromechanics framework for modeling transverse cracks in fiber reinforced composites was outlined. The ECI model was used at the matrix-matrix and fiber-matrix subcell interfaces in an HFGMC model. The evolution of transverse cracks was demonstrated using two simple RUCs: a single fiber, and two diagonally arranged fibers. Both RUCs exhibit crack nucleation at the fiber boundaries and crack bridging between adjacent fibers.

The current model used to capture interfacial discontinuities is explicitly dependent on time. Current progress is being made towards including debonding governed by traction-separation laws into MAC/GMC via the DCZM formulation. The use of DCZM formulations for interfacial debonding and fracture would incorporate fracture toughness and cohesive strength characteristics of interfaces into the prediction of transverse crack evolution. Validation of these features will include a comparison to analogous finite element models that employ DCZM elements at the solid element boundaries.

\section{References}

${ }^{1}$ Cox, B. and Yang, Q., "In quest of virtual tests for structural composites," Science, Vol. 41, 2006, pp. 6526-6546.

${ }^{2}$ Kouznersova, V., Brekelmans, W. A. M., and Baaijens, F. P. T., "An approach to micro-maro modeling of heterogenerous materials," Comput. Mech., Vol. 27, 2001, pp. 37-48.

${ }^{3}$ Bednarcyk, B. A. and Arnold, S. M., "A framework for performing multiscale stochastic progressive failure analysis of composite structures," Proceedings of the 2006 ABAQUS User's Conference, 23-25, May 2006.

${ }^{4}$ Talreja, R., "Multi-scale modeling in damage mechanics of composite materials," J. Mater. Sci., Vol. 41, 2006, pp. 6800-6812. 2008.

${ }^{5}$ Kwon, Y. W., Allen, D. H., and Talreja, R., editors, Multiscale Modeling and Simulation of Composite Materials and Structures, Springer,

${ }^{6}$ Talreja, R., "Multi-scale modeling of composite solids with damage," Proceedings of the American Society for Composites Twenty-third Technical Conference, 9-11, Sept. 2008.

${ }^{7}$ Pineda, E. J., Waas, A. M., Bednarcyk, B. A., and Collier, C. S., "A novel, multiscale high fidelity progressive damage and failure modeling approach for laminated fiber reinforced composites," Proceedings of the American Society for Composites Twenty-third Technical Conference, 7-10, Apr. 2008.

${ }^{8}$ Talreja, R., "Transverse cracking and stiffness reduction in composite laminates," J. Composite Materials, Vol. 19, 1985, pp. $355-275$.

${ }^{9}$ Aboudi, J., Mechanics of Composite Materials: A Unified Micromechanical Approach, Elsevier Amsterdam, 1991.

${ }^{10}$ Paley, M. and Aboudi, J., "Micromechanical analysis of composites by the generalized cells model," Mechanics of Materials, Vol. 14, 1992, pp. 127-139.

${ }^{11}$ Bazant, Z. P., "Nonlocal damage theory based on micromechanics of crack interactions," J. Eng. Mech. - ASCE.

${ }^{12}$ Prat, P. C. and Bazant, Z. P., "Tangential stiffness of elastic materials with systems of growing or closing cracks," J. Mech. Phys. Solids, Vol. 45, No. 4, 1997, pp. 611-636.

${ }^{13}$ Aboudi, J., Pindera, M.-J., and Arnold, S. M., "Higher-order theory for functionally graded materials," Composites Part B - Engineering, Vol. 30, 1999, pp. 777-832.

${ }^{14}$ Aboudi, J., Pindera, M.-J., and Arnold, S. M., "Higher-order theory for periodic multiphase materials with inelastic phases," Int. J. Plast., Vol. 19, 2003, pp. 805-847.

${ }^{15}$ Bednarcyk, B. A., Aboudi, J., and Arnold, S. M., "Micromechanics modeling of composite materials subjected to multiaxial progressive continuum damage," 50th AIAA/ASME/ASCE/AHS/ASC Structures, Structural Dynamics, and Materials Conference, 4-7 May 2009.

${ }^{16}$ Bednarcyk, B. A. and Arnold, S. M., "MAC/GMC 4.0 User's Manual - Keywords Manual," Nasa tm-2002-212077/vol2, 2002.

${ }^{17}$ Bednarcyk, B. A. and Arnold, S. M., "MAC/GMC 4.0 User's Manual - Example Problems Manual,” Nasa tm-2002-212077/vol3, 2002.

${ }^{18}$ Bednracyk, B. A., Arnold, S. M., Aboudi, J., and Pindera, M.-J., "Local field effects in titanium matrix composites subject to fiber-matrix debonding," Int. J. Plast., Vol. 20, 2004, pp. 1707-1737.

${ }^{19}$ Song, S. J. and Waas, A. M., "An energy based model for mixed mode failure of laminated composites," AIAA Journal, Vol. 33, No. 4, 1995.

${ }^{20}$ Xie, D. E., Salvi, A., Sun, C. E., Waas, A. M., and Caliskan, A., "Discrete cohesive zone model to simulate static fracture in 2-D triaxially braided carbon fiber composites," J. Compos. Mater, Vol. 40, No. 22, 2006.

${ }^{21} \mathrm{Xie}$, D. and Waas, A. M., "Discrete cohesive zone model for mixed-mode fracture using finite element analysis," Engineering Fracture Mechanics, Vol. 73, No. 13, 2006, pp. 1783-1796.

${ }^{22}$ Gustafson, P. A. and Waas, A. M., "The influence of adhesive constitutive parameters in cohesive zone finite element models of adhesively bonded joints," Int. J. Solids Struct., 209, pp. 2201-2215.

${ }^{23}$ Ranatunga, V., Bednarcyk, B. A., and Arnold, S. M., "Modeling delamination in composites via continuum interfacial displacement discontinuities," 50th AIAA/ASME/ASCE/AHS/ASC Structures, Structural Dynamics, and Materials Conference, 4-7 May 2009.

${ }^{24}$ Jones, J. P. and Whittier, J. S., "Waves at flexibly bonded interfaces," J. Appl. Mech., Vol. 34, 1967, pp. 905-909.

${ }^{25}$ Hashin, Z. and Rotem, A., "A fatigue failure criterion for fiber reinforced composite materials," J. Composite Materials, Vol. 7, 1973, pp. $448-464$

${ }^{26}$ Sanford, R. J., Principles of Fracture Mechanics, Pearson Education, Inc., 2002. 